\title{
The Hydraulic Characteristics of the Reactor - Clarifier Including Ripple Contact Mass Particles
}

\author{
Yuri L. Skolubovich, Mikhail S. Soppa, \\ Natalya V. Sineeva and Alexey Y. Skolubovich* \\ Novosibirsk State University \\ of Architecture and Civil Engineering (Sibstrin) \\ 113 Leningradskay, Novosibirsk, 630008, Russia
}

Received 08.12.2016, received in revised form 20.01.2017, accepted 02.02.2017

The paper considers the model of hydrodynamic processes in the reactor - clarifier that uses filtering technology in a fluidized layer. Dynamic load ripple particles lead to a substantial increase in clean suspensions from the impurities. Simulation allows to determine the loss of pressure in different parts of the structure. The free flow of power component costs associated with the hydrostatic pressure and viscous friction forces in laminar flow. In the fluidized layer these costs are due to the grain loading parameters: diameter, density, porosity. On the basis of the proposed model of self-oscillatory movement of grain loading obtained relations for estimation of particle ripple effect on the value of head loss. Therefore necessary to average power in increments of hydraulic gradient is established. Analysis of hysteresis phenomena in various design modes spend, due to the fact that the height of the weighted layer is not a unique function of supplying treated water rate. It is shown that the occurrence of the hysteresis loop can be connected with the capture of the self-oscillation frequency of movement of the contact mass particles.

Keywords: simulation, fluidized layer, the grain loading, the self-oscillations, the loss of pressure, the hysteresis.

Citation: Skolubovich Yu.L., Soppa M.S., Sineeva N.V., Skolubovich A.Y. The hydraulic characteristics of the reactor clarifier including ripple contact mass particles, J. Sib. Fed. Univ. Eng. technol., 2017, 10(1), 36-43. DOI: 10.17516/1999494X-2017-10-1-36-43.

(C) Siberian Federal University. All rights reserved

* Corresponding author E-mail address: rector@sibstrin.ru,m2011soppa@gmail.com, ief@sibstrin.ru 


\title{
Гидравлические характеристики
}

\section{реактора-осветлителя с учетом пульсаций частиц контактной массы}

\author{
Ю.Л. Сколубович, М.С. Соппа, \\ Н.В. Синеева, А.Ю. Сколубович \\ Новосибирский государственный \\ архитектурно-строительный университет (Сибстрин) \\ Россия, 630008, Новосибирск, Ленинградская, 113
}

В статье рассмотрена модель гидродинамических процессов в реакторе-осветлителе, использующем технологию фильтрации в псевдоожиженном слое. Динамические пульсации частии загрузки приводят к существенному увеличению очистки суспензий от примесей. Моделирование позволило определить потери напора на различных участках конструкции. В компоненте свободного течения затраты мощности связаны с гидростатическим давлением и с силами вязкого трения в ламинарном течении. На участке псевдоожиженного слоя эти затраты обусловлены параметрами зерен загрузки: диаметром, плотностью, пористостью. На основе предложенной модели автоколебательного движения зерен загрузки получены соотношения для оценки влияния пульсаций частии на величину потерь напора. Установлена связь необходимой осредненной мощности с приращением гидравлического уклона. Проведен анализ гистерезисных явлений при различных режимах работы конструкиии, обусловленных тем, что высота взвешенного слоя не однозначная функиия скорости подачи очищаемой воды. Показано, что возникновение петли гистерезиса может быть связано с захватом частоты автоколебательного движения частии контактной массы.

Ключевые слова: моделирование, псевдоожиженный слой, зерно загрузки, автоколебания, потеря напора, гистерезис.

\section{Введение}

Установки с использованием псевдоожиженного слоя получили широкое распространение в различных промышленных и технологических областях, связанных с адсорбцией, катализом, теплообменом, фильтрационной очисткой и др. [1]. Возникающие в слое динамические пульсации частиц способствуют увеличению поверхности их контакта с потоком, активируют диффузию и, в частности, существенно увеличивают скорость очистки суспензий от примесей [2]. Важная задача - описание, расчет и моделирование различных явлений, реализующихся в псевдоожиженном слое. Описание резонансных режимов динамических пульсаций, инициированных внешним вынуждающим воздействием, дано в статье [3], в [4] рассмотрено протекание процессов релаксационных, разрывных автоколебаний в неоднородных слоях. В работе [5] на основе исследования математической модели колебательного движения зерен загрузки установлены основные (в том числе энергетические) параметры нестационарного процесса, получена оценка периода колебаний. Предложенный в данной статье подход применен к расчетам гидравлического сопротивления для компонент реактора-осветлителя, изучены гистерезисные характеристики течений в псевдоожиженном слое. 


\section{Определение гидравлического сопротивления}

Рассмотрим гидравлическую схему реактора-осветлителя (рис. 1). Введем обозначения: $L$ - полная высота столба жидкости в реакторе, $L_{1}$ - высота взвешенного слоя загрузочной массы, $R_{1}, R_{2}-$ внутренний и внешний радиусы канала.

Свободное течение на участке $\left[L_{1}, L\right]$ длиной $L_{2}=L-L_{1}$ описывается в гидравлическом приближении. Следствие уравнения неразрывности на этом участке имеет вид $\rho v_{1} S=\rho v_{2} S$, поэтому скорость постоянна, $v=$ const, и скоростной напор $h_{v 2}$ равен нулю. Из уравнения Бернулли определяется статический напор $h_{z 2}=\Delta p_{z 2} / \rho \mathrm{g}=L_{2}$.

При скорости $v=U=0,0028 \mathrm{~m} / \mathrm{c}$ течение на участке $\left[L_{1}, L\right]$, очевидно, носит ламинарный характер. Площадь поперечного сечения канала равна $S_{0}=\pi\left(R_{2}^{2}-R_{1}^{2}\right)$, поэтому эффективный диаметр канала определяется по формуле

$$
D_{e}=\sqrt{4 S_{0} / \pi}=2 \sqrt{R_{2}^{2}-R_{1}^{2}},
$$

а число Рейнольдса можно оценить значением $\mathrm{Re}_{2}=\frac{\rho v D_{e}}{\mu}$, где $\mu-$ коэффициент вязкости. Определим потерю напора $h_{t 2}$ за счет трения на участке $\left[L_{1}, L\right]$. Она выражается через коэффициент сопротивления (вязкого трения) $\lambda$ с использованием формулы Вейсбаха-Дарси $h_{t 2}=\lambda \frac{L_{2} v^{2}}{D_{e} 2 g}$, где $\lambda=75 / \operatorname{Re}_{2}$, отсюда $h_{t 2}=75 \frac{L_{2} \mu v}{D_{e}^{2} 2 \rho g}$.

Таким образом, на участке $\left[L_{1}, L\right]$ потеря напора имеет вид

$$
h_{2}=h_{t 2}+h_{z 2} \text {. }
$$

Определим величину потери напора $h_{s 1}$ на участке $\left[0, L_{1}\right]$, в столбе взвешенного псевдоожиженного слоя, следуя методике, изложенной в [2]. Воспользуемся известными формулами Д. Минца для коэффициента сопротивления ч и числа Рейнольдса:

$$
\psi=\frac{\Delta p_{1} m_{s}^{3} d}{L_{1} \rho v^{2} 6\left(1-m_{s}\right) \alpha}
$$

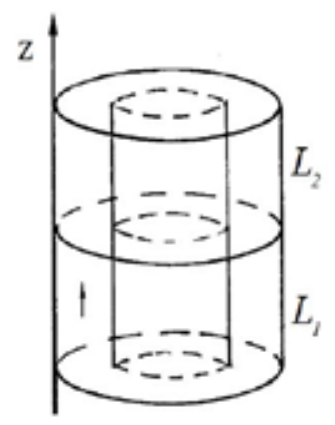

Рис. 1. Схема канала течения 


$$
\operatorname{Re}_{1}=\frac{\rho v d}{6 \mu\left(1-m_{s}\right) \alpha}
$$

где $m_{s}$ - пористость загрузки; $d$ - диаметр зерен; $\alpha$ - коэффициент формы частиц загрузки. Величину коэффициента сопротивления можно также оценить на основании теории подобия: $\psi=\frac{A}{\mathrm{Re}_{1}}$, где параметр $A$ имеет представление: $A=1,5 \alpha^{2}$.

Подставляя в формулу (2) зависимость $\Delta p=\rho g h_{s 1}$, приходим к соотношению

$$
h_{s 1}=\psi \frac{12\left(1-m_{s}\right) \alpha L_{1}}{m_{s}^{3} d}\left(\frac{v^{2}}{2 g}\right) .
$$

Отсюда, в частности, можно определить гидравлический уклон данного участка канала $i_{1}=\frac{h_{s 1}}{L_{1}}$, что вполне согласуется с численными оценками этой характеристики по формуле Козени-Кармана [2]. К этому слагаемому добавляются потери напора $h_{z 1}=\frac{\Delta p_{z 1}}{\rho g}=L_{1}$ на преодоление силы тяжести.

В результате проведенного анализа получаем, что суммарные потери напора по длине реактора-осветлителя вычисляются по формуле

$$
h=h_{t 2}+h_{z 2}+h_{s 1}+h_{z 1}+\Delta h,
$$

где $\Delta h-$ потери напора на границе раздела в канале при $z=L_{1}$.

Для вычисления расхода энергии за единицу времени в виде требуемой мощности насосов по обеспечению функционирования системы используем соотношение $h=\Delta p /(\rho g)$. Умножим числитель и знаменатель дроби на элемент объема $\Delta V=v \Delta t S$, соответствующий приращению времени $\Delta t$ :

$$
h=\frac{\Delta p \Delta V}{\rho g \Delta V}=\frac{F v \Delta t S}{S \rho g \Delta V} .
$$

Учитывая, что $\rho g \frac{\Delta V}{\Delta t}=Q$ - это массовый расход жидкости через сечение канала, а $F v=P-$ мощность, получаем требуемое выражение

$$
P=Q h
$$

\section{Влияние нестационарного движения частиц взвешенного слоя на величину потери напора}

В работе [5] предложен подход к моделированию нестационарных характеристик движения частиц в псевдоожиженном слое контактной массы. Движение зерен в вертикальном канале описывается обыкновенным дифференциальным уравнением 


$$
m \frac{d u}{d t}=-m g+\rho_{v} V g-6 \pi \mu R(u-U)
$$

где $U$ - скорость несущего потока очищаемой воды; $m$ - масса частицы; $R$ - радиус частицы; $V=(4 / 3) \pi R^{3}$ - ее объем; $\rho_{v}$ плотность воды; $F_{c}=6 \pi \mu R(u-U)$ сила сопротивления вязкой жидкости по закону Стокса; $(u-U)$ - относительная скорость.

На участке движения вверх начальные условия имеют вид

$$
u(t=0)=u_{0}, x(t=0)=0 .
$$

Для последующего участка движения в противоположном направлении для функции $u\left(t_{1}\right)$ ставится начальное условие

$$
u\left(t_{1}=0\right)=-u\left(t_{0}\right), t_{1}=t-t_{0} .
$$

Интегрирование этих уравнений позволяет получить решение в виде

$$
\begin{aligned}
& u(t)=\left(u_{0}-p\right) e^{-q t}+p, \\
& x(t)=x_{0}+t p+\left(u_{0}-p\right)\left(1-e^{-q t}\right) / q,
\end{aligned}
$$

где $p=U-\left(m g-\rho_{v} V g\right) /(6 \pi \mu R) ; q=6 \pi \mu R / m$.

На рис. 2 приведен фазовый портрет автоколебательного процесса для модуля скорости частицы.

Во время движения на частицу за счет эффектов вязкости действует сила $f_{\tau}=-6 \pi \mu R(u-U)$. Модуль мощности взаимодействия вязкого потока с частицей при обтекании оценивается выражением

$$
P(t)=\left|f_{\tau}(u-U)\right|=6 \pi \mu R(u-U)^{2} .
$$

За один период автоколебаний силами вязкого взаимодействия будет совершена работа

$$
A_{\tau}=A_{\tau 1}+A_{\tau 2}
$$

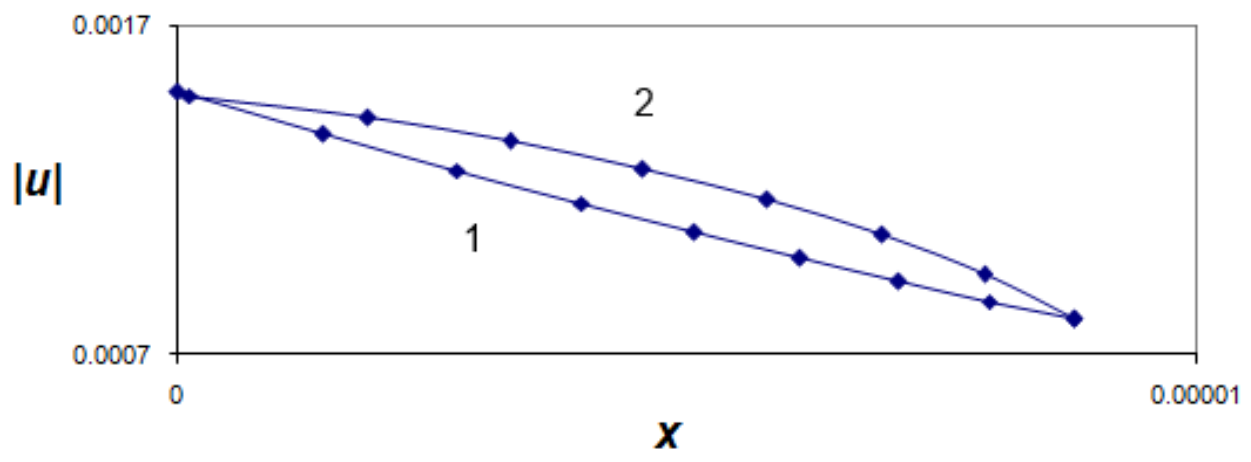

Рис. 2. Фазовый портрет автоколебаний 
Здесь

$$
A_{\tau 1}=\int_{0}^{T_{1}} P(t) d t=\int_{0}^{T_{1}} 6 \pi \mu R(u-U)^{2} d t
$$

- работа на участке движения вверх, а

$$
A_{\tau 2}=\int_{0}^{T_{2}} P\left(t_{1}\right) d t_{1}=\int_{0}^{T_{2}} 6 \pi \mu R(u+U)^{2} d t_{1}
$$

- работа, совершенная при движении вниз.

Раскрывая подынтегральные выражения, получим

$$
\begin{aligned}
& A_{\tau 1}=6 \pi \mu R\left(U^{2} T_{1}-2 U \int_{0}^{T_{1}} u d t+\int_{0}^{T_{1}} u^{2} d t\right), \\
& A_{\tau 2}=6 \pi \mu R\left(U^{2} T_{2}+2 U \int_{0}^{T_{2}} u d t_{2}+\int_{0}^{T_{2}} u^{2} d t_{2}\right) .
\end{aligned}
$$

Принимая во внимание представление для скорости частицы (5), приходим к вспомогательным формулам

$$
\begin{gathered}
\int_{0}^{T_{1}} u d t=\int_{0}^{T_{1}}\left(u_{0}-p\right) e^{-q t} d t+p T_{1}=\left(u_{0}-p\right)\left(1-e^{-q T_{1}}\right) / q \\
\int_{0}^{T_{1}} u^{2} d t=\int_{0}^{T_{1}}\left(\left(u_{0}-p\right) e^{-q t}+p\right)^{2} d t=\left(u_{0}-p\right)^{2}\left(1-e^{-2 q T_{1}}\right) /(2 q)+ \\
+2\left(u_{0}-p\right) p\left(1-e^{-q T_{1}}\right) / q+p^{2} T_{1}
\end{gathered}
$$

Аналогичные выражения для $\int_{0}^{T_{2}} u d t_{1}$ и $\int_{0}^{T_{2}} u^{2} d t_{1}$ справедливы и на втором участке движения.

Подстановка найденных соотношений в $(7,8)$ позволяет согласно $(6)$ вычислить $A_{\tau}$.

Перейдем к оценке величины $\Delta E$ - суммарной потери энергии за цикл для всех частиц контактной массы. Количество частиц во взвешенном слое можно приближенно определить по формуле, учитывающей возможное их размещение в объеме, зависящее от поперечного сечения частицы:

$$
N_{c h}=\frac{0.8 S L_{1}}{2 \pi R^{3}}=\frac{0.53 \mathrm{~V}}{V_{c h}},
$$

где $V$ - объем взвешенного слоя, а $V_{c h}$ - объем частицы загрузки. Таким образом, $\Delta E=N_{c h} A_{\tau}$. Осредненная по периоду колебаний расходуемая мощность находится по формуле

$$
P_{s r}=\frac{N_{c h}\left(\int_{0}^{T_{1}} P(t) d t+\int_{0}^{T_{2}} P\left(t_{1}\right) d t_{1}\right)}{T_{1}+T_{2}}=\frac{\Delta E}{T_{1}+T_{2}} .
$$


Учитывая соотношение (4), связывающее потерю напора с массовым расходом жидкости, получаем зависимость

$$
h_{k o l}=P_{s r} / Q
$$

которая соответствует выражению для приращения гидравлического уклона

$$
i_{k o l}=P_{s r} /\left(L_{1} Q\right)
$$

где $L_{1}$ - высота взвешенного столба контактной массы.

\section{Гидродинамический гистерезис в псевдоожиженном слое}

Предложенная и рассмотренная выше физико-математическая модель установки способствует уточнению и дополнительному описанию гистерезисных характеристик поведения высоты столба взвешенного слоя при различных режимах работы конструкции.

При увеличении скорости подачи очищаемой жидкости ожижение столба контактной массы происходит не сразу, а лишь когда скорость превысит некоторый порог, равный $U_{p s}$ (рис. 3). Дальнейшее возрастание скорости приводит к заметному росту высоты слоя $L_{1}$, в свою очередь потеря напора стабилизируется на определенном значении. Этот процесс длится до достижения верхней критической скорости $U_{k r}$, характеризующейся массовым уносом частиц загрузки потоком. Если теперь начать постепенно уменьшать скорость подачи воды, то высота взвешенного слоя уменьшается уже по иной зависимости, образуя при этом гистерезисную петлю.

В рамках предлагаемой модели ветвление гидродинамического решения объясняется следующим образом. При одной и той же скорости подачи воды возможны два режима автоколебаний. Первый - с более высокой частотой колебаний $\omega_{1}$, соответствующей меньшей высоте взвешенного слоя, и второй режим - с меньшей частотой колебаний $\omega_{2}$, реализующийся при увеличенной высоте слоя $L_{1}$. В нелинейной теории колебаний этот эффект известен под названием «захват» частоты [6]. Расчеты по формулам (7-9) показывают, что на поддержание автоколебаний (с учетом поперечных пульсаций) требуется не более 0,5 \% всех потерь напора, обеспечивающего фильтрацию через слой загрузочной контактной массы.

Если в процессе дальнейшего уменьшения скорости подачи очищаемой воды происходит фильтрация через слой при скорости меньшей $U_{p s}$, то высота слоя после его первоначального псевдоожижения остается несколько выше, чем у полностью неподвижного слоя. Отмеченное

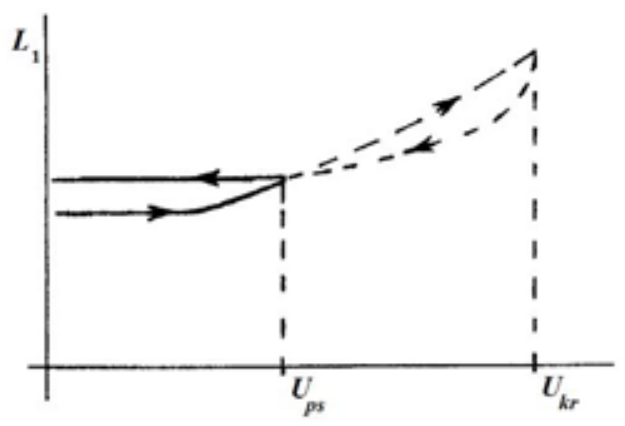

Рис. 3. Высота взвешенного слоя. Петля гистерезиса 
увеличение высоты объясняется действием сил сцепления между неподвижными зернами. Данное обстоятельство приводит к понижению (подтвержденному и в эксперименте) примерно на $\Delta p \approx 7$ \% гидравлического сопротивления при указанном режиме работы установки.

\section{Заключение}

С использованием предложенной ранее математической модели автоколебаний зерен загрузки в системе для очистки воды проведены уточненные исследования величины гидравлического сопротивления на различных компонентах установки. В результате интегрирования зависимостей для фазовых переменных получены оценки дополнительных потерь для поддержки нестационарных пульсаций. Показано, что возникновение петли гидродинамического гистерезиса может быть связано с захватом частоты автоколебаний при различных режимах фильтрования с применением технологии псевдоожиженного слоя.

\section{Список литература}

[1] Айнштейн В.Г. Баскаков А.П. Берг Б.В. и др. Псевдоожижение. М.: Химия, 1991. 398 c. [Aynshteyn V.G. Baskakov A.P. Berg B. V. et al. Fluidization. M.: Chemistry, 1991, 398 p. (in Russian)]

[2] Войтов Е.Л. Сколубович Ю.Л. Подготовка питьевой воды из поверхностных источников с повышенным природным и антропогенным загрязнением. Новосибирск: НГАСУ (Сибстрин), 2010. 217 c. [Voitov E.L. Skolubovich Y.L. Drinking water from surface sources with a high natural and anthropogenic pollution. Novosibirsk: NSUACE (Sibstrin), 2010. 217 p. (in Russian)]

[3] Малков А.А. Малыгин А.А. Перспективы модифицирования дисперсных материалов методом молекулярного наслаивания с использованием колебательного изменения давления газовой фазы. Известия РГПУ им. А.И. Гериена. Современная техника и технология, 2007, 7(26), 127-136. [Malkov A.A. Malygin A.A. Perspectives the modification of disperse materials by atomic layer deposition using the oscillating pressure changes of the gas phase. News RGPU. A.I. Herzen. Modern equipment and technology. 2007, 7(26), P. 127-136 (in Russian)]

[4] Тупоногов В.Г. Рыжков А.Ф. Баскаков А.П. Обожин О.А. Релаксационные автоколебания в псевдоожиженном слое. Теплотехника и аэромеханика, 2008, 15(4), 643-657 [Tuponogov V.G. Ryzhkov A.F. Baskakov A.P. Obozhin O.A. Relaxation oscillations in a fluidized layer, Heat and Aeromechanics, 2008, 15(4), 643-657].

[5] Сколубович Ю.Л. Соппа М.С. Синеева Н.В. Моделирование нестационарных характеристик движения частиц контактной массы в реакторе-осветлителе. Изв. вузов. Строительство, 2015, 7, 38-43. [Skolubovich Y.L. Soppa M.S. Sineeva N.V. Simulation of non-stationary characteristics of the motion of the contact mass of particles in the reactor-clarifier, Proceedings of the universities. Building, 2015, 7, 38-43 (in Russian)]

[6] Хаяси Т. Нелинейные колебания в физических системах. М.: Мир, 1968, 432 с. [Hayashi T. Nonlinear vibrations in physical systems. M., Mir, 1968, 432 p. (in Russian)] 